\title{
SIALOGRAPHY IN DIAGNOSIS
}

\author{
By S. S. Rose, M.B., F.R.C.S. \\ First Assistant, Department of Surgery, University of Manchsster
}

\section{History}

The injection of radiopaque media to supplement a clinical opinion has long been regarded as a valuable aid to diagnosis. The importance of sialography, however, although emphasized repeatedly by Payne, seems to have escaped general attention and comparativelv few papers have been published on this subject.

The first account of a sialogram carried out as a diagnostic measure appeared in 1925 when Barsony described a method of outlining the parotid duct, using 20 per cent. potassium iodide. This medium, however, proved to be so irritant that he abandoned the technique. A year later the introduction of lipiodol provided a further stimulus to this form of investigation, but, in spite of its widespread adoption, it was not until I93 I that R. T. Payne published the first of his classical series of papers on the subject. Using a glass pipette of the type shown in Fig. I, he injected $\frac{1}{2}$ to $\mathrm{I} \mathrm{ml}$. of lipiodol very slowly into the appropriate duct orifice until discomfort was complained of. An X-ray was taken immediately with the instrument in situ. The main points in the technique were that the apparatus was light and so less likely to slip out of position during the subsequent manipulations of the patient's head, and that there was no detachable needle to be forced off the end of the syringe. The latter difficulty had been previously experienced on account of the force necessary to inject the extremely viscid medium. This method of sialography gave a clear picture of the main duct system, and often extended into the finer ducts and acini when the injection was complete. The use of neohydriol fluid, however, has obviated most of the early technical difficulties, as its low viscosity allows greater ease and speed of injection with the minimum of effort.

It has recently been argued that water-soluble radiopaque media should be used on account of their low viscosity and because they show no tendency to break up into globules. It has been found, however, that such media as viskiosol run quickly out of the gland and even when the sialogram is carried out with the needle in position, the resulting picture is poor.

\section{Technique}

The present technique has been arrived at by consideration of all the factors involved and has $\vec{\omega}$ proved satisfactory in a series of 93 sialograms, $\frac{\rho}{\circ}$ without any untoward effects. Routine straight $\mathrm{X}$-rays are taken before sialography.

An ordinary $5 \mathrm{ml}$. record syringe is used with $\stackrel{\sim}{\circ}$ a blunt number $I$ or 2 intravenous needle angled ${ }^{\omega}$ slightly in the upper third of its shaft to facilitate or its introduction into the duct orifice. A lock-on needle is sometimes advocated (Barsky and Silber- 0 man, 1932) but has not been found necessary when the less viscous media are used.

There is usually no difficulty in finding the $\frac{0}{0}$ opening of Stensen's duct, opposite the site of the second upper molar tooth, by eversion of the cheek between thumb and forefinger. If it is not in $\vec{G}$ mediately obvious, massage over the gland and. duct will produce a rapid flow of saliva from orifice which dilates to two or three times its normal size. The opening of Wharton's duct, however, is situated on a mobile papilla which must be stabilized before insertion of the blunt $\frac{\AA}{\Phi}$ cannula. This may be achieved by the use of a fine non-toothed dissecting forceps which seizes $\frac{0}{3}$ the papilla immediately above the opening and gently renders taut the line of the duct. The tongue is pressed upwards and forwards against the hard palate. The use of $\frac{1}{2}$ per cent. hydro- $\frac{3}{3}$ chloric acid solution may sometimes be necessary to produce a flow of saliva and a supply is kept in readiness on the sialography tray. It is often $\delta$ necessary to dilate the duct orifice before insertion of the cannula and, for this purpose, modified 을 lacrimal duct dilators are used (Fig. 2). In cases of duct stenosis, preliminary duct dilatation is essential before sialography, but over-dilatation will give a false picture on X-ray and may allow $N$ too rapid emptying of the gland. The degree of $N$ dilatation, therefore, should only be that necessary to allow easy introduction of the cannula.

The volume of fluid injected in this series has been rather more than that advocated by other $\frac{\mathscr{D}}{\mathbb{D}}$ authors, namely $\mathrm{I} \frac{1}{2}$ to $2 \mathrm{ml}$. This does not increase $\stackrel{\mathcal{D}}{?}$ the discomfort, providing that the injection is not 0 made too quickly, and it is found that, although discomfort may appear after I $\mathrm{ml}$. has been in- 


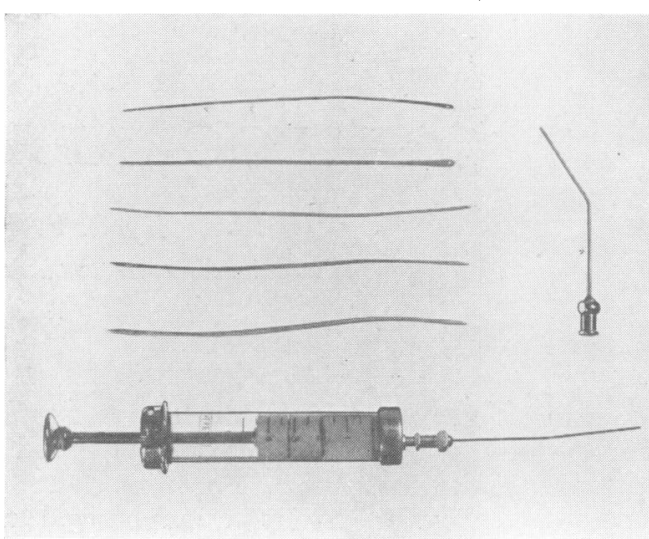

Fig. 2.- Syringe, needles and dilators.

Fig. I.-Glass pipette of type recommended by Payne (1931).
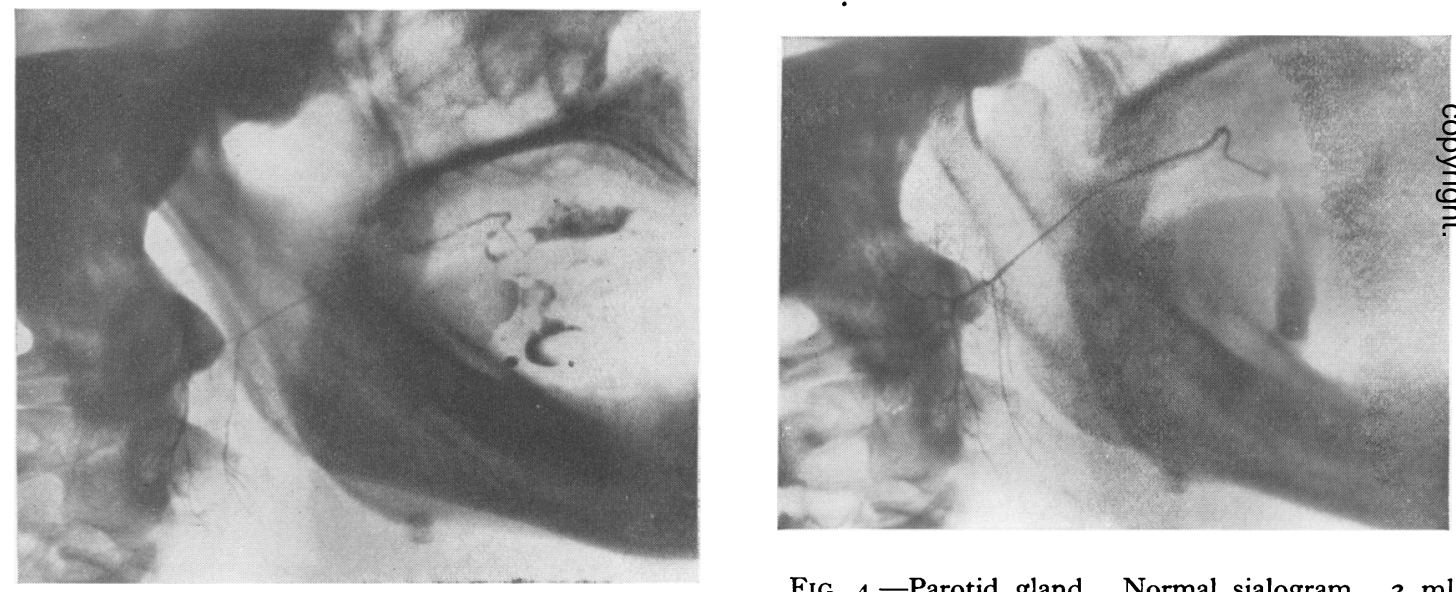

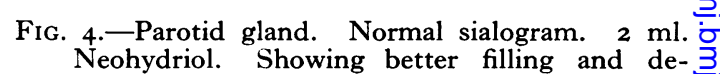
finition. This is the small largely retromandibular type of gland.

FIG. 3.-Parotid gland. Normal sialogram. I $\mathrm{ml}$.
Neohydriol.

jected, it does not increase appreciably as the injection continues. By this modification of technique it has been found possible to fill the gland so adequately that not only is the duct system outlined but an outline of the surrounding gland parenchyma is also visualized. The accurate delineation of the gland is of particular value in the diagnosis of space occupying lesions of the salivary gland, for the lesion may be either too small or too peripheral to distort the duct anatomy.

The needle is inserted with the patient seated opposite the surgeon while an assistant supports the head. Care must be taken in cannulating the

parotid duct so that the needle is not inserted so fars that it is occluded by the end of the buccal part? of the duct, and it is found that a distance of $2 \mathrm{~cm} . \mathrm{N}$ is adequate. A finger is held over the duct orifice during the injection and this is replaced by a lint $N$ swab which is removed immediately before the $\mathrm{X}$-ray is taken. This ensures a minimum of losso into the mouth with subsequent complication of the radiographic appearance. A good picture may be obtained up to 20 minutes after the injection? There is, therefore, no need for the rather difficulto manipulation required with the cannula in situ

The routine views taken are as follows: 


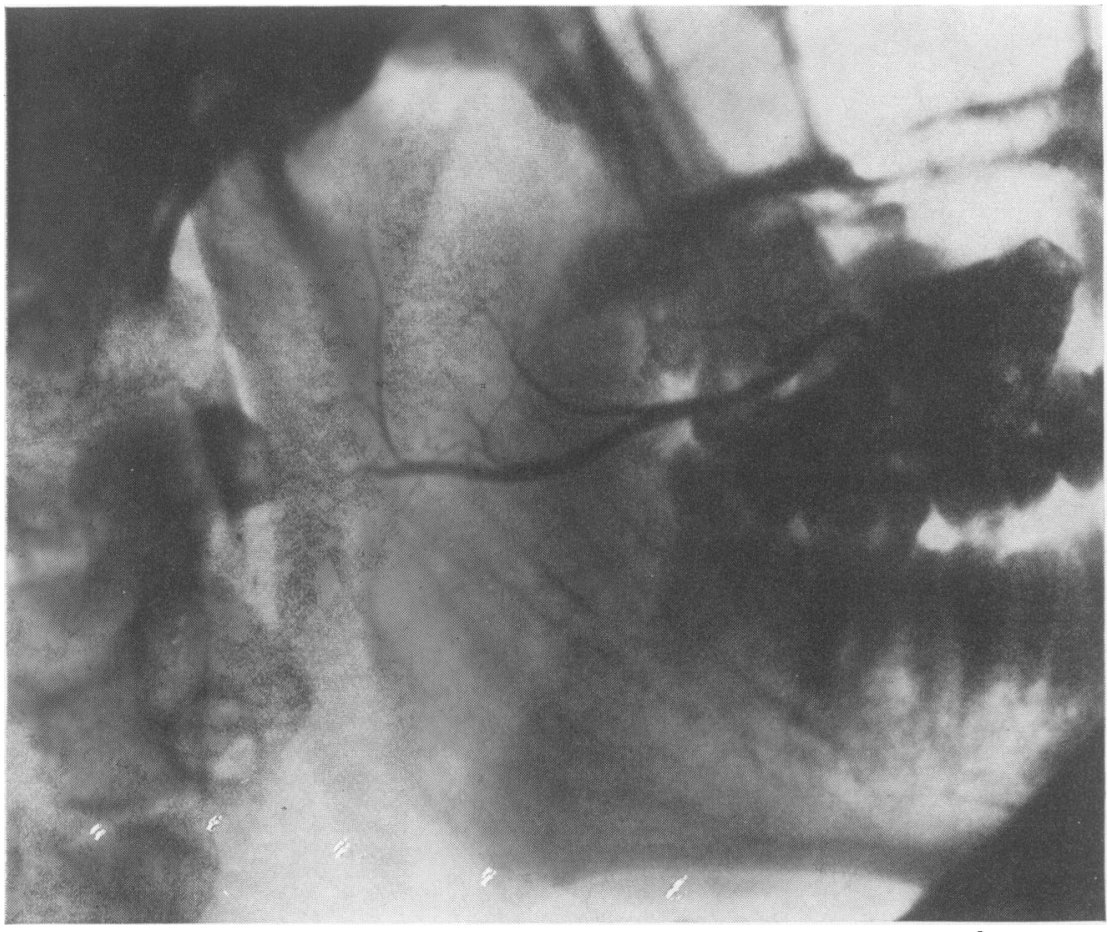

FIG. 5.-Parotid gland. Normal sialogram. Large type of gland with well marked socia parotidis. The gland extends well forward on to the masseter.

\section{Parotid Gland}

Anteroposterior and true lateral films usually give all the required information. The oblique view serves only to distort the normal anatomy and is not carried out as a routine procedure. It may, however, be used in conjunction with the lateral view to elucidate spatial relationships if these are somewhat obscure.

\section{Submandibular Gland}

The most useful views here are the lateral and oblique lateral, but a basal view is occasionally helpful in establishing the extra- or intraglandular position of a filling defect. The insertion of a dental occlusion film in the floor of the mouth gives information about the intraoral part of the duct but is now only used when specially indicated, for example in the exact localization of a stone in this region.

\section{The Normal Sialogram}

Normal sialograms achieved by this technique are illustrated in Figs. 3, 4, 5 and 6 . Figs. 3 and 4 show the different degrees of filling obtained with injections of $1 \mathrm{ml}$. and $2 \mathrm{ml}$. respectively in the same gland. The main duct is uniformly fine and $\frac{\tilde{\Phi}}{\mathbb{D}}$ there is no evidence of terminal dilatation. Fig. $7 \mathrm{a}$ 을 shows a diagrammatic representation of the parotid $\overrightarrow{\hat{O}}$ duct system. Experience with the normal parotid sialogram shows that the duct anatomy is reasonably constant. Fig. 7 b similarly shows a diagram-matic representation of the submandibular glando showing the right-angled bend where the duct runs down from the floor of the mouth into the $\frac{5}{3}$ gland substance. It is seen that the submandibular ducts on the whole are much shorter and wider $\mathrm{O}$ than those of the parotid, an anatomical fact which may have a bearing on the relative frequency of $\frac{}{2}$ recurrent infection in the latter gland.

\section{Clinical Application}

The type of for or sialography is the recurrent parotid swelling, usu lly after a straight film has revealed now evidence of a calculus. The incidence of calculi? in the parotid is as low as 2 per cent. of salivaryco stones and most cases of recurrent parotid swelling $\mathbb{\complement}$ fall into one of the following groups:

(I) Recurrent pyogenic parotitis.

(2) Simple duct obstruction.

(3) Secondarily infected duct obstruction. 
FIG. 7a.-Diagram of parotid sialogram. There are usually three ascending ducts as well as the duct of the socia, if present, and one or two descending ducts depending on the size of the gland. Several small retromandibular ducts drain the deep part of the gland.

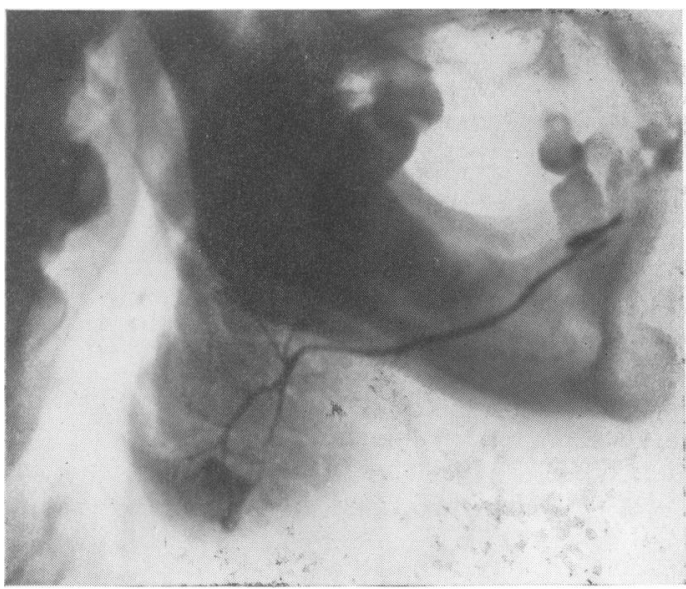

Fig. 6.-Submandibular gland. Normai sialogram. The right-angled bend where the main duct turns down into the gland is well marked. A posterior sublingual duct is seen entering the main duct.

\section{(i) Recurrent Pyogenic Parotitis}

This interesting and by no means uncommon condition was first described by Payne (1933), who demonstrated the 'bronchiectatic' type of terminal dilatation of the parotid duct. He also showed that the commonest infecting organism in this condition was the Streptococcus Viridans, a finding repeated in this series. Other organisms reported have been the Streptococcus Pyogenes,

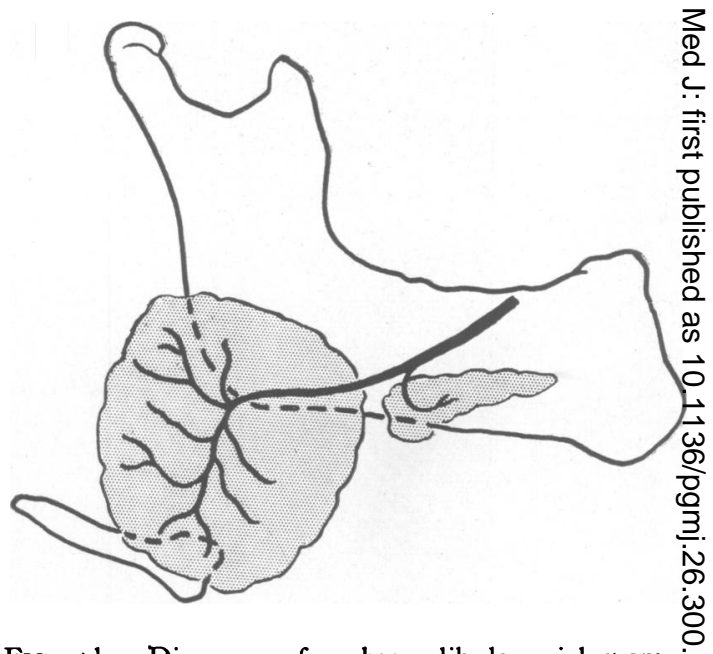

Fig. 7b.-Diagram of submandibular sialogram. ơ The subsidiary duct descending from the angle of $N$ the jaw to join the angle of the main duct is very 으 constant.

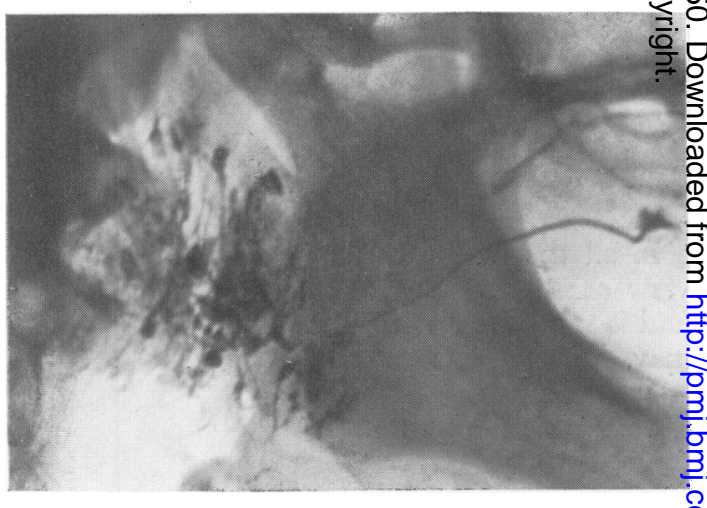

FIG. 8.-Early recurrent pyogenic parotitis. S. viridans infection. Lateral view showing 0 moderate sialangiectasis.

B. Proteus, Pneumococcus, organisms of Vincent's $N$ angina and even atypical coliform bacilli.

\section{Streptococcus Viridans Infection}

This infection characteristically occurs in women during the fourth and fifth decades. Only? 3 out of 24 in the present series were men. $\square$

The patient first notices an aching pain in fronto of or just below the tragus and this is normallyo 


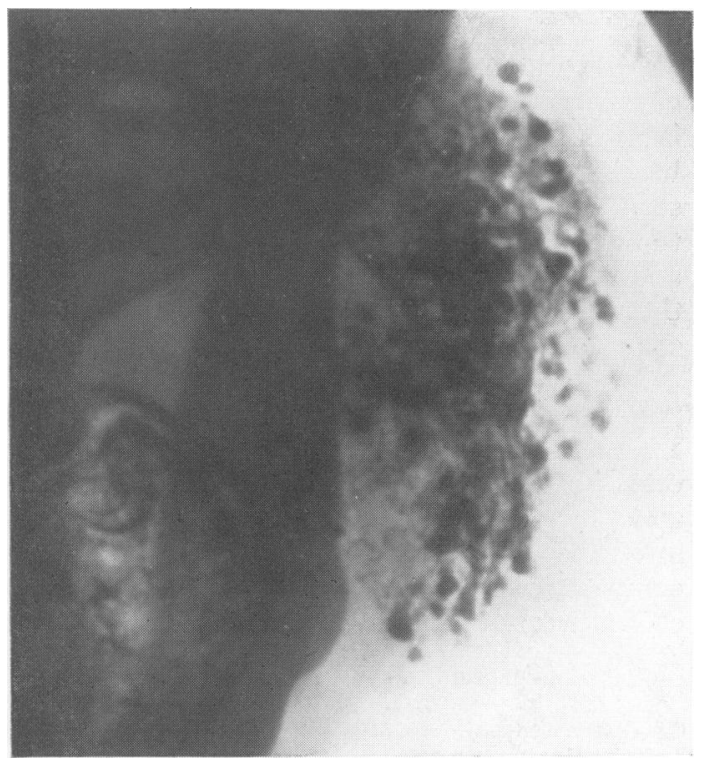

FIG. 9.-Advanced recurrent parotitis. S. viridans infection. Antero-posterior view, showing marked sialangiectasis.

accompanied by a slight diffuse parotid swelling. The onset may be preceded by a febrile illness, although more often no predisposing cause can be found. The patient may volunteer the information that she is subject to attacks of dryness of the mouth, particularly when undergoing a period of mental stress. The earlier attacks may last only a few hours, with remissions up to six months or a year. They may begin in one gland and be followed by attacks of greater severity in the opposite gland. At first the swelling subsides completely between attacks, leaving only a slight thickening. Later it persists and the gland may become palpable throughout its whole extent, the surface becoming smoothly nodular and of a rubbery consistency. If there is only a localized infection a firm nodule may be produced which is difficult to distinguish clinically from a salivary tumour. The history and sialogram will serve to differentiate between these conditions. The saliva during the attacks has particulate globules of mucopus containing desquamated cells and bacteria, giving the characteristic ' snowstorm' effect (Payne, 1938). The constant discharge of infected saliva into the mouth causes the patient to complain of an unpleasant taste and occasionally the end of an attack may be announced by a sudden heavy mucopurulent discharge.

In recurrent parotitis there is no close relationship between the pain and food, but there is often a history of an attack commencing at the beginning of a meal, particularly in the earlier cases.

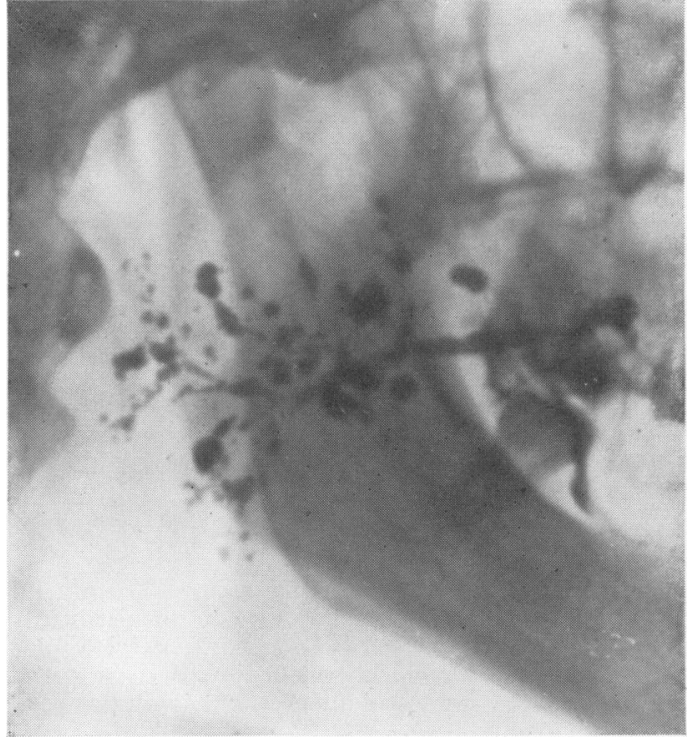

FIG. 10.-Recurrent parotitis. Pneumococcal infection. Marked dilatation of duct and gross sialangiectasis. Lateral view.

As the disease progresses, food has less and les8 effect and it may be that by the time the patien is referred for treatment, the early relationship te meals has been forgotten.

The Sialogram. In the early case, the terminal dilatation may be confined to one area alone or there may be scattered isolated dilatations (Fig. 8). The duct itself shows no abnormality. The gland parenchyma is usually well outlined and no anatomical abnormalities are revealed.

In the late case of $\mathrm{S}$. viridans infection the typical picture of sialangiectasis is seen (Fig. 9). There is a saccular dilatation of the terminal ducts and acini, and the main ducts become 'beaded.' This appearance results from alternate dilatation and narrowing of the duct, and is an indication of the duration of the infection. It is also noteworthy that the shadow of the gland parenchyma is either very faint or non-existent. This is partially due to lack of filling in parts of the gland which are occluded by inflammatory exudate or by fibrosis, and partially due to the reservoir-like action of the dilated duct system.

Pneumococcal Recurrent Parotitis (Payne, 1940)

This condition deserves special mention on account of several features which distinguish it 0 both clinically and sialographically from the $S$. viridans infection. The attacks of pain and swelling are severe and the degree of general disturbance of health is considerable. Abscess formation may occur by the fusion of several pockets

$\overrightarrow{0}$ . 


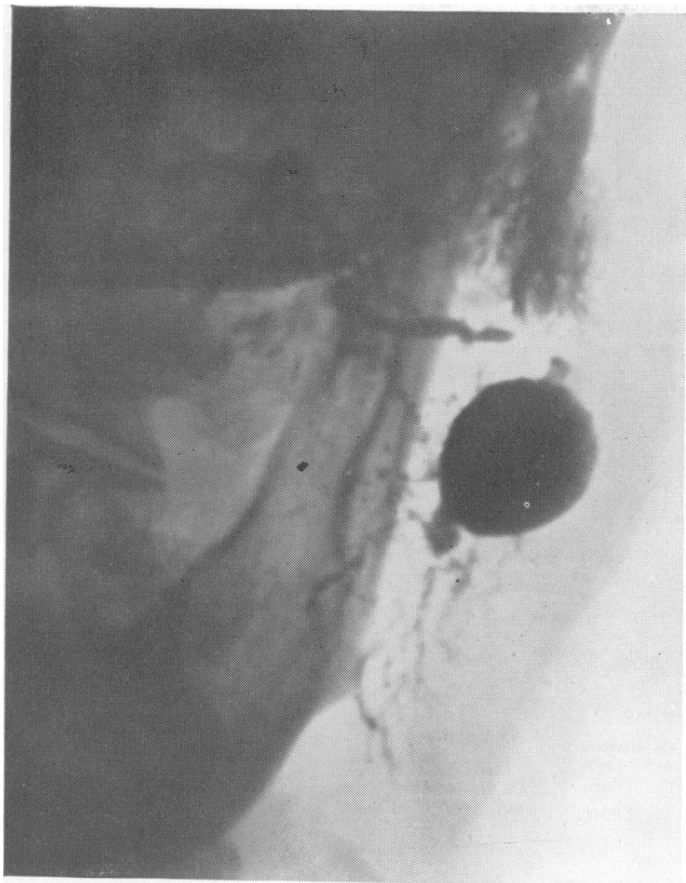

FIG. II.-Parotid abscess. Pneumococcal infection. Antero-posterior view.

of pus which may be discharged into the parotid duct, thus terminating the infection. The saliva is purulent, often with a characteristic greenish tinge, and on culture a heavy growth of pneumococci is readily obtained.

The sex incidence of pneumococcal parotitis is curious in that in the present series 8 cases out of 9 have been males.

The Sialogram. Marked disorganization of the terminal duct system is evidenced by the gross degree of dilatation seen, with no evidence of a

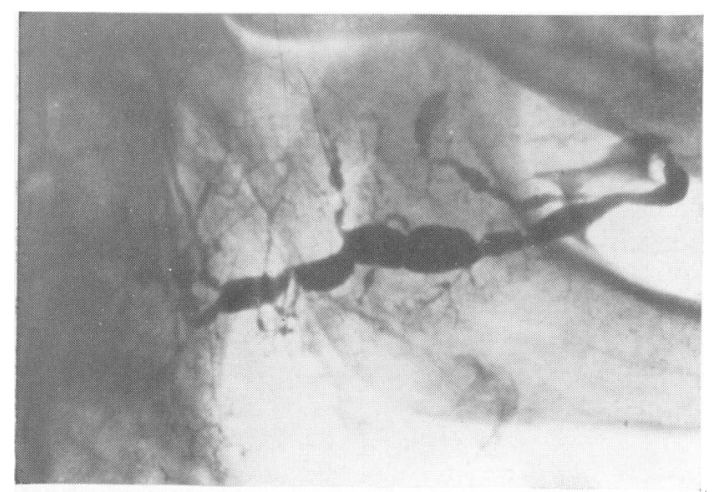

FIG. 14.-Secondarily infected duct obstruction. See text. gland outline (Fig. 10). The main duct in this case is much wider than normal with periods constrictions along its length. Occasionally thạs is so marked that a 'sausage-string' appearane has been noted (Ollerenshaw and Rose, 1950) seen in Fig. I4. It is evident that the reaction of the duct to infection is one of alternate strictum and dilatation and that this is the same whether the primary cause is obstruction at the orifice $\mathbb{\mathbb { P }}$ a long-standing recurrent pyogenic parotitis. \&

Parotid Abscess. The late case of pneumococclit parotitis may give rise to the picture seen in Fig I I, that is, of a localized parotid abscess. Fives cases in the present series showed this appearange and all were pneumococcal in origin. The histogy in all these cases was of long-standing, recurrent, comparatively severe attacks of pain and swelling each attack resembling the acute post-operative typis of parotitis. They differed from the latter only the absence of the extreme general prostration encountered in the acute post-operative infection. In all these cases the abscess discharged sporn taneously into the duct, and external drainage was not required.

\section{Simple Duct Obstruction}

This condition is more common than recurents pyogenic parotitis and in its uncomplicated form is a separate entity. However, when complicated by infection, the obstructive type bears a cFose clinical resemblance to the inflammatory lesion and may only be differentiated by a careful historis and by sialography.

It has been shown above that in recurrent parơ titis there is no predisposing obstructive cause i $\overrightarrow{\vec{a}}$ the main duct. In the early case the abnorma findings are confined to the terminal ducts an acini, the main duct being of normal size. Such dilatation as occurs in the later stages is behing multiple inflammatory strictures. It will be shown. below that quite a different state of affairs exist in simple duct obstruction.

\section{Etiology}

The two main types of duct obstruction are produced by similar mechanisms and are depen dent on an abnormality of mastication following of accompanying a dental derangement. They mafs be divided anatomically into papillary and buccało according to the portion of the duct involved.

The papillary duct obstruction is caused boy trauma to the papilla of Stensen's duct ans though it usually occurs in the presence of ile

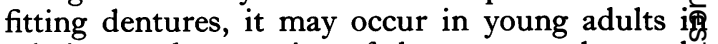
relation to the eruption of the upper molar teeth.

In the younger age groups an inflamed and tender gum will produce a deviation from the normal chewing habit. This may cause injury to 


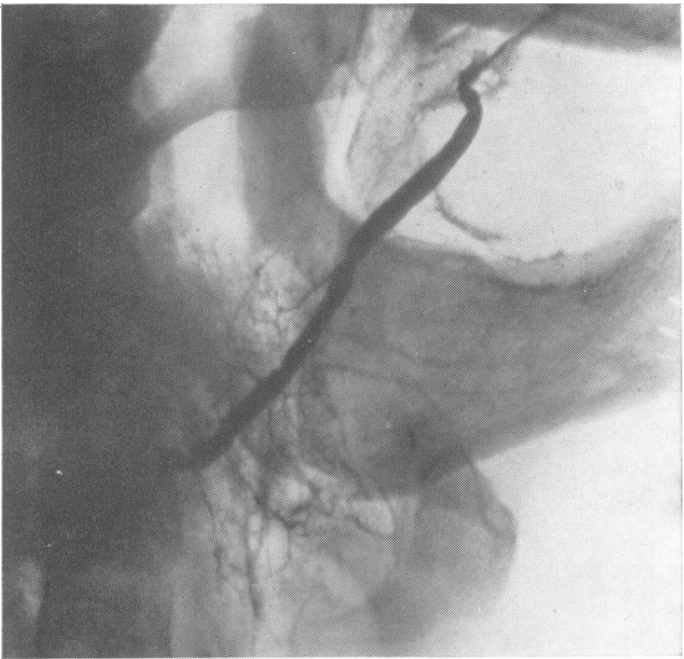

FIG. I 2.-Simple duct obstruction. Papillary type. Oblique lateral view.

Stensen's papilla with subsequent oedema and ulceration. When the original inflammation has settled down, fibrosis may develop at the duct orifice and stenosis ensue. The cases in this series occurred between the ages of twenty and twenty-five.

In the older age group a similar repeated trauma to the duct orifice is seen, but here the cause is an ill-fitting denture. It is often found that the progressive alveolar absorption occurring with advancing age results in the upper denture becoming loose and undergoing an abnormal degree of upward and downward movement with mastication. The papilla is repeatedly traumatized as the denture rides up in the alveobuccal sulcus and ulceration may occur. This again may result in the papillary type of obstruction. More rarely, where the whole of the inner side of the cheek over the line of the duct is involved, the fibrosis may spread backwards and the buccal type of obstruction develops. The latter always occurs in the older age groups in the presence of dentures, although even here the papillary duct obstruction is the more common.

\section{Clinical Picture}

The patient presents with a diffuse parotid swelling accentuated by meals, and complains of discomfort rather than pain. The history is usually short, as the persistent nature of the swelling causes advice to be sought during the first attack.

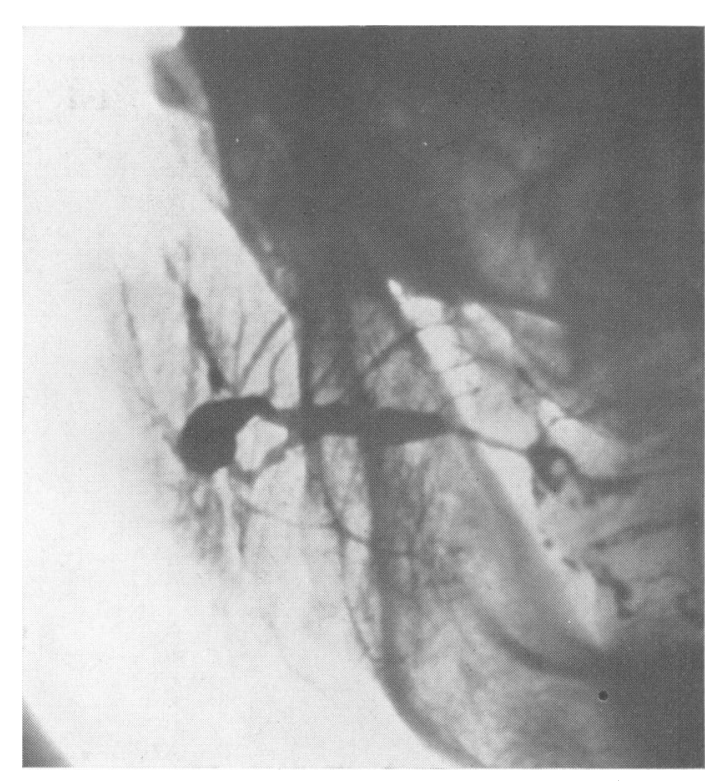

FIG. I3.-Simple duct obstruction. Buccal type Antero-posterior view.

The gland is diffusely palpable, but the edges are imperceptible, and there is none of the nodularity felt in the infective cases. Pressure over the gland will give rise to a sluggish flow of clear saliva from a rigid-looking orifice situated on a hypertrophied papilla. The saliva is sterile on culture and shows no increase of mucoid or cellular content.

It is these cases of duct obstruction which are often cured by the sialogram itself, as it is necessary to dilate the duct as a preliminary procedure. Recurrent parotitis, however, requires a routine of at least six consecutive daily injections of the duct with one-half to three-quarters of a million units of penicillin. This treatment has proved to be uniformly satisfactory in 18 cases reviewed over a two-year period.

\section{The Sialogram}

Fig. 12 shows a papillary duct obstruction in a man $4 \mathrm{I}$ years of age. The main duct is dilated, narrowing down to a fine point at its oral end. The gland itself is not involved and there is no evidence of sialangiectasis. The papilla was hypertrophied and exuded sterile saliva on pressure over the gland. His dentures were 12 years old and the upper set had become increasingly mobile.

Fig. I3 shows a buccal duct obstruction in a woman 59 years of age. Here the stricture extends up the duct to the point where it perforates the buccinator muscle. Beyond the 


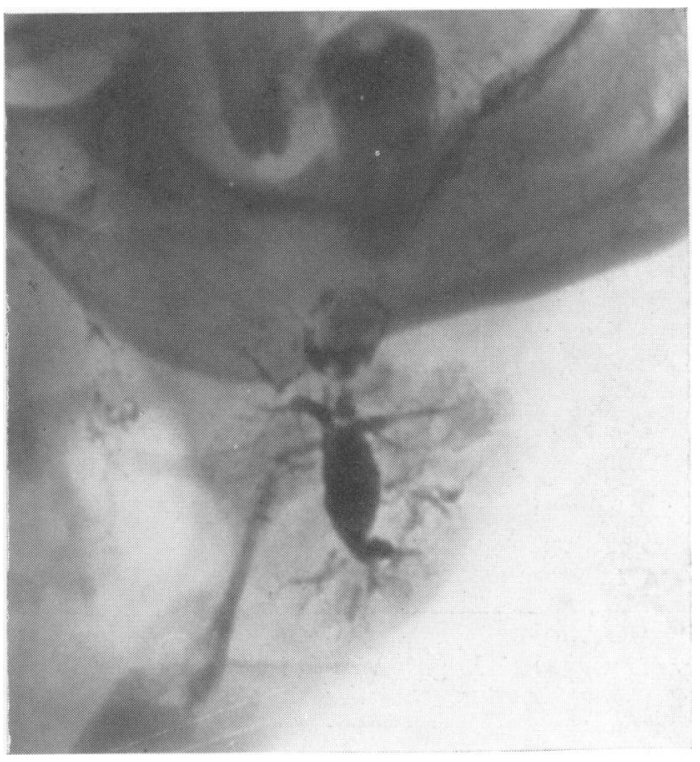

FIG. 15.-Submandibular calculus. The calculus lies in the deep part of the duct after it turns down into the gland. Dilatation of the main duct behind this, but no sialangiectasis.

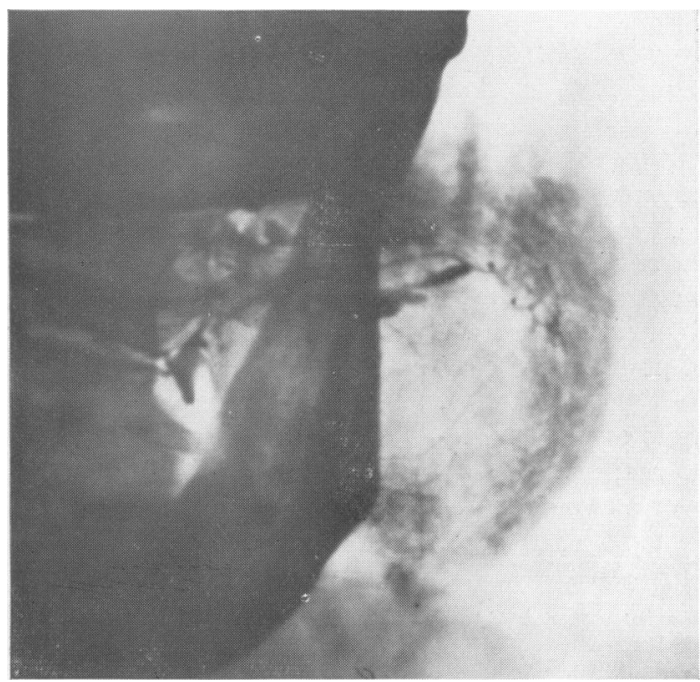

FIG. 17.- Mixed parotid tumour. There is a central filling defect with upward displacement of Stensen's duct.

obstruction the duct is dilated in a somewhat irregular manner, indicating a degree of secondary infection. There is, however, no evidence of involvement of the terminal ducts and the gland pattern appears to be normal. The dentures in this case had been worn for 21 years. Six years

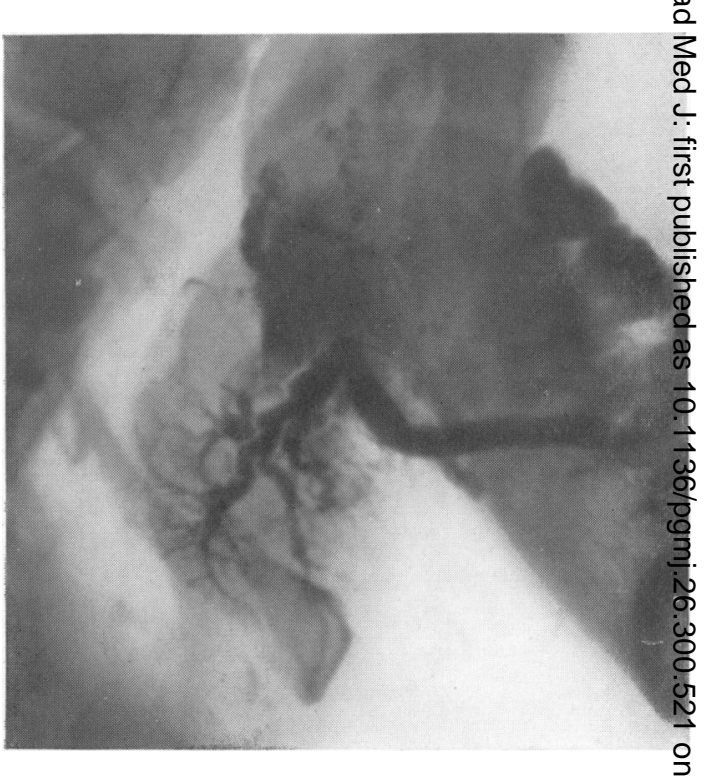

Fig. 16.-Efects of terminal obstruction of Wharton's duct. Obstructed for two months on ans off. Note dilatation of duct without sialangiec $\frac{1}{0}$ tasis.

previously the lower set had been discardedo they were loose, and the unsupported upper showed a wide range of both vertical and laters movement. There was ulceration in both alveos buccal sulci extending on the right side forward along the line of the duct. This relationshio could be readily demonstrated by insertion of $\stackrel{\Phi}{\Phi}$ fine probe.

(3) Duct Obstruction with Secondary INFECTION

It has been pointed out that on occasion it extremely difficult to distinguish between a secors. darily infected duct obstruction and a long-stand ing recurrent parotitis. The buccal type of obstruction, in particular, is prone to secondaro infection and in the case shown in Fig. $14 \mathrm{~S}$. vir? dans was obtained on culture.

The history of these cases is longer than that the simple types and there tend to be more pai and thickening of the gland. The relationship to food is constant, however, and there is evidenee of trauma to the papilla or the cheek overlying the duct. The dentures are ill-fitting and may haves been worn for many years. Occasionally the dentures have never fitted properly and the lesion may be fully developed within six months.

The Sialogram (Fig. 14) shows that the duct hơs borne the brunt of the infection and is seen to bo dilated and strictured. Some of the minor ducs share in the dilatation, but the parenchyma of the 


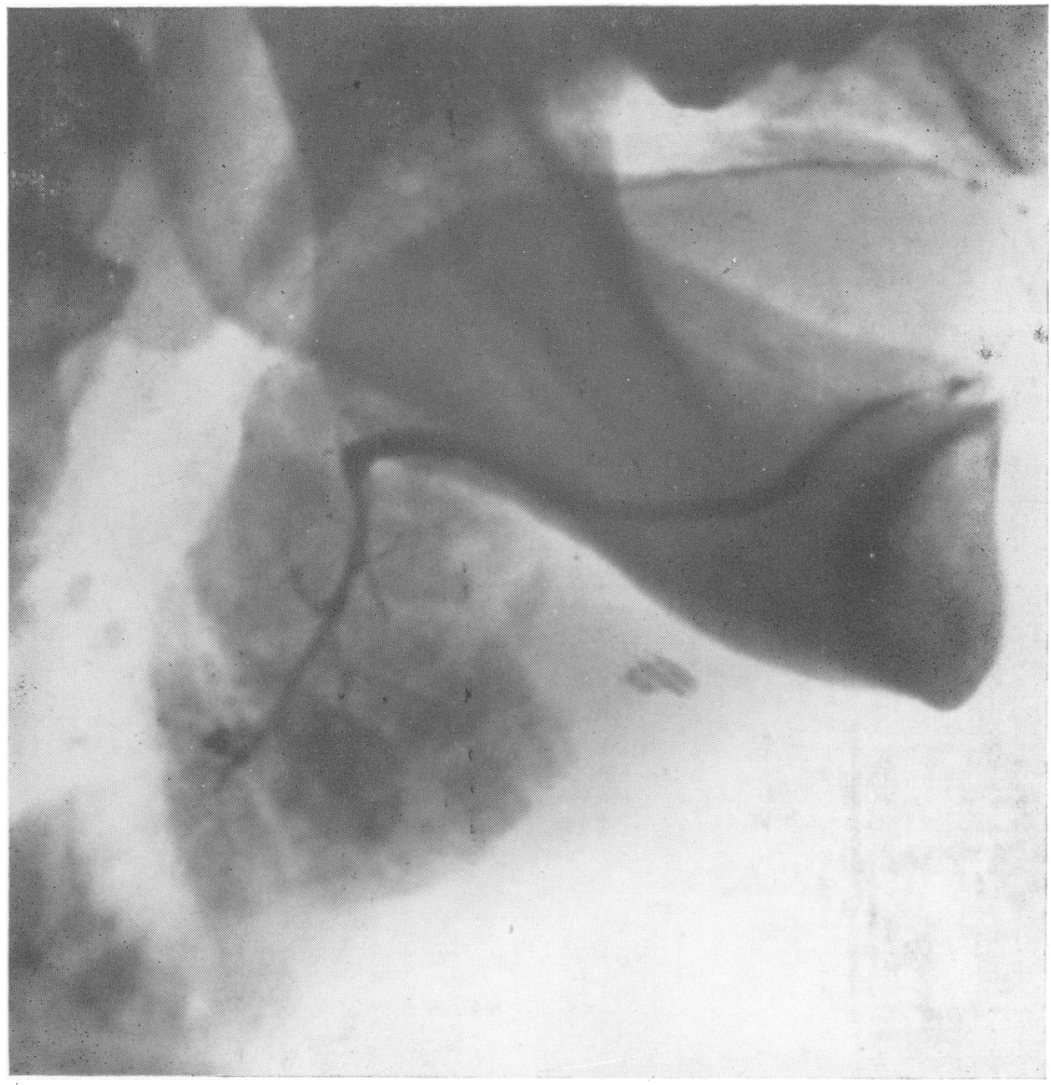

FIG. 18.-Submandibular filling defect.

gland is not involved and there is no acinar abnormality. The infecting organism in this case was the $\mathrm{S}$. haemolyticus.

As the majority of duct obstructions are sterile, it is felt that it is justifiable to place these infected cases in a separate category.

\section{Salivary Calculi}

When salivary calculi are considered, the submandibular gland claims all the attention. It is argued that it is unnecessary to employ sialography in the diagnosis of salivary calculi as the plain $\mathrm{X}$-ray will establish their presence. This argument is perfectly justifiable, but must be accepted with certain reservations. Firstly, Feuz (1932) has pointed out that up to 20 per cent. of salivary calculi are radiotranslucent and, secondly, a knowledge of the exact whereabouts of the stone may be essential before treatment is decided upon. A calculus lying in the floor of the mouth may be easily removed transorally, but if it should lie at or behind the point at which the main duct turns down into the gland, satisfactory removal is only achieved by sacrifice of the submandibular gland itself. Fig. 15 is a sialogram performed to ascertain the exact position of a salivary calculus. It was impossible to say from the straight X-ray whether 3 the stone was intraglandular or not, and bidigital palpation of the floor of the mouth failed to localize 3 the calculus. Fig. 15 shows the stone to be lying $\delta$ in the deep part of the duct, and indicates the line $₹$ of treatment.

Fig. 16 is a sialogram taken after removal of a 욱 calculus from the termination of Wharton's duct and shows the degree of dilatation the duct system undergoes. The absence of sialangiectasis again $\sigma$ indicates the essentially non-obstructive nature of $N$ the recurrent salivary infections. This is further $\mathcal{N}_{\mathrm{W}}$ suggested by comparing the scant incidence of ${ }^{\omega}$ recurrent infection of the submandibular gland with the frequent occurrence of calculi.

\section{Salivary Tumours}

It is sometimes difficult to decide whether a ${ }^{\circ}$ swelling in the parotid or submandibular region $\vec{D}$ is actually in the gland concerned or is superficial $\stackrel{?}{\stackrel{\odot}{\odot}}$ 


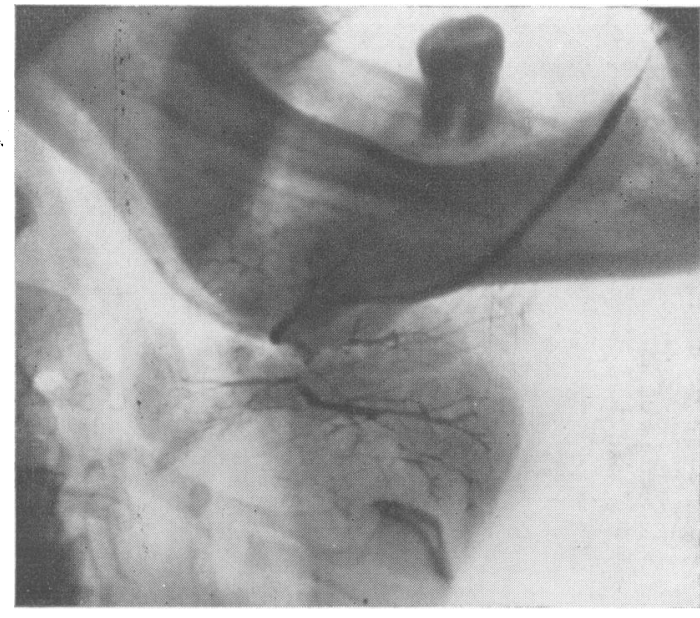

Fig. 20a.-Submandibular filling defect. A large tumour is displacing the duct upwards.

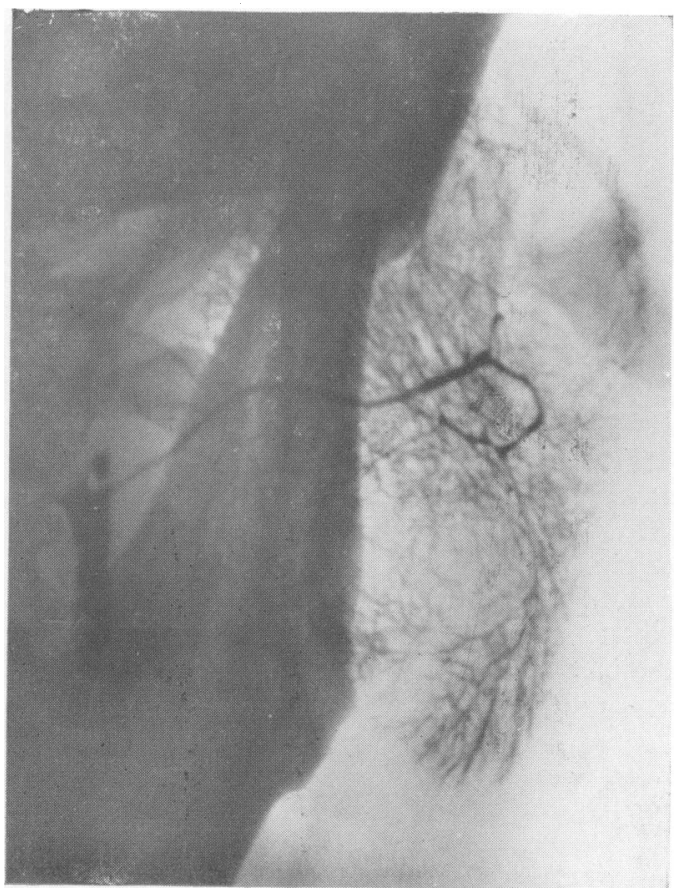

FIg. 19.-Parotid filling defects. There are two filling defects one above and one below the main duct. These were intra-parotid tuberculous lymph glands.

to it. The sialogram may be valuable in determining the relationships of such a swelling to the gland parenchyma (Leroux, 1947) particularly when the latter is well outlined by adequate filling of the gland (Ollerenshaw and Rose, 1950). There is no doubt that interpretation of the sialogram in

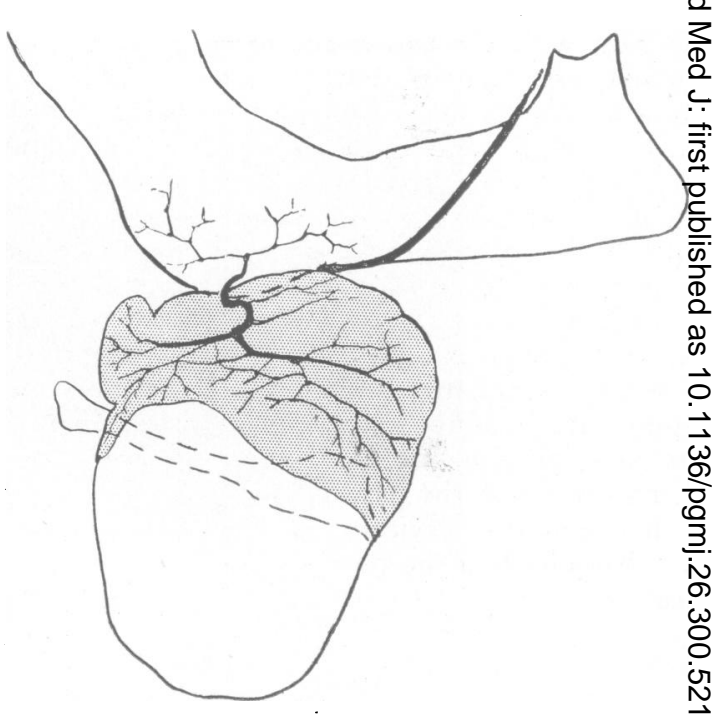

FIG. 2ob.-Diagram of 2oa, to show tumour outline.음

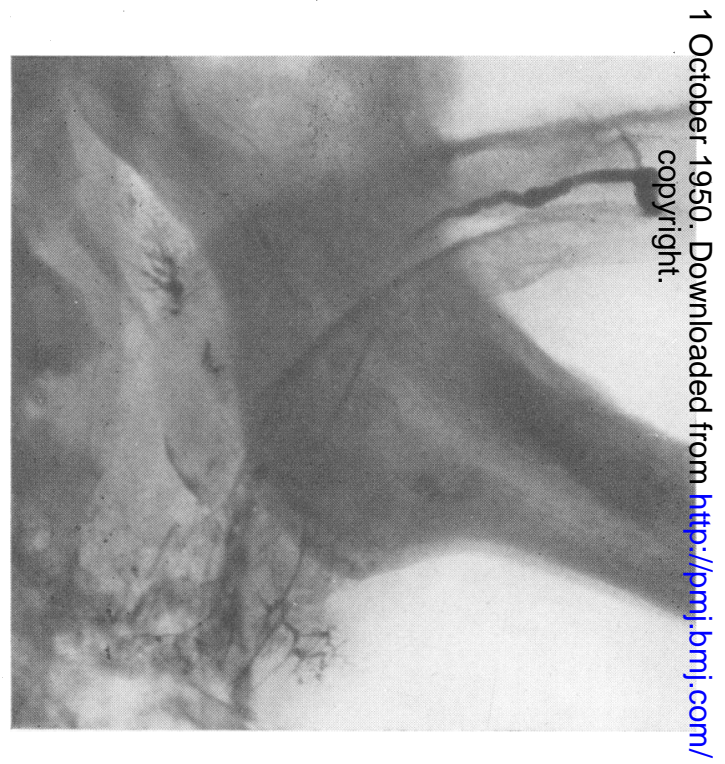

FIG. 21.-Malignant parotid tumour. Gross destruc- 음 tion of gland outline and irregularity of duct pattern.

cases of tumour may be difficult and it is only byo combination of a careful analysis of the pictures with a knowledge of the normal appearances that a diagnosis can be made. The various points that require special attention are illustrated by the sialograms described under the following headings

The Filling Defect

This is the common finding with tumours and depends for its presence on a space occupyings lesion unfilled by the radiopaque medium. Theे 
area shows as a comparative translucency corresponding with the soft tissue swelling (Fig. $i$ ). An extrinsic tumour may produce no effect on the gland, the aialogram being normal, or it may cause a filling lefect in the profile of the gland (Fig. 18). Her the anterosuperior portion of the gland has not outlined and the defect corresponds with the site of the swelling. In the centre of the translucency there is a small area of calcification; a diagnosis of tuberculous lymphadenitis was therefore made and was confirmed at operation. Similarly, lymphatic swellings in the preauricular region are readily distinguished from parotid tumours, and in one case the presence of two filling defects in the parotid gland in conjunction with an enlarged cervical gland enabled a diagnosis of tuberculosis of the parotid lymph glands to be made with confidence (Fig. 19).

\section{Distortion of the Duct Anatomy}

It has been stated that the sialogram shows a constant duct anatomy, and any gross deviation from the normal pattern must be accounted for. The main duct may be pushed out of position and be seen running over an obvious intraglandular tumour or two adjacent subsidiary branches may be widely separated by an interposed neoplasm. In most neoplasms of the salivary glands, sialographic localization depends on a combination of duct distortion and local filling defect (Fig. 20a). Reference to the accompanying diagram (Fig. 2ob) shows how in this case the submandibular duct is displaced upwards and compressed into a small area, while the gland below it is replaced by a large spherical translucent area. This was a large 'mixed' salivary tumour which had been present for nine years.

Two sialograms in the present series, one of which is shown in Fig. 21, caused special comment on account of the peculiar distortion seen, the smaller ducts seeming to fill in an irregular manner, leaving many areas completely unfilled. There was also little evidence of glandular outline and the whole appearance suggested a gland in- 气 volved by an infiltrative process. Clinically, the pain, hardness and fixity of the swelling strongly suggested a malignant parotid tumour (Fursten- $\subseteq$ burg, 1948). It has been suggested that a malig- $\overrightarrow{\vec{s}}$ nant tumour will give a characteristic picture, but the comparative rarity of the lesion has so far given little opportunity for sialographic study. We were unable, therefore, on the appearances shown to do more than suggest a diagnosis of malignancy. Further experience with correlation of patho- $\omega$ logical findings may enable a more dogmatic $\overrightarrow{0}$ diagnosis to be made.

\section{Summary}

The history and technique of sialography is 3 discussed and the author's modifications described. N

The normal parotid and submandibular sialograms are demonstrated and emphasis is laid on the constancy of the duct anatomy.

The appearance of the sialogram in cases of recurrent pyogenic parotitis, parotid duct obstruc- 은 tion, submandibular calculus and salivary tumours is described. The sialogram is correlated with the clinical picture in each case.

The duct obstructions are classified anatomically with reference to the sialogram and their etiology is briefly discussed.

Sialography is invaluable in the diagnosis of duet obstruction and recurrent infection and may useful in the diagnosis of intra- and extraglandular swellings.

\section{Acknowledgment}

Acknowledgments are due to Professor A. M. Boyd, who has stimulated and encouraged this line of study, to Dr. E. D. Gray and the staff of the Department of Radiology of Manchester Royal Infirmary for their help and willing co-operation, to the Department of Medical Photography for providing the illustrations, and to the Honorary Surgeons who made all possible by referring their cases of parotid swelling for investigation.

\section{BIBLIOGRAPHY}

BARSKY, A. J., and SILBERMAN, H. (1932), Ann. Surg., 95, 46 BARSONY, T. (1926), Klin. Wschr., 4, 2500.

FEUZ, J. (1932), Schweiz. Med. Wschr., 62, 666.

FURSTENBURG, A. C. (1948), F. Amer. Med. Ass., 136, I.

LEROUX, G. F. (1947), f. de Radiol. et D'Elect., 28, 85.

OLLERENSHAW, R. G. W., and ROSE, S. S. (1950), Brit. $\mathcal{f}$. Radiol., in pres.

PAYNE, R. T. (1931), Brit. F. Surg., 19, 142.

PAYNE, R. T. (1933), Lancet, I, 342.

PAYNE, R. T. (1938), Prac. Roy. Soc. Med., 31, 398.

PAYNE, R. T. (1940), Brit. Med. F., 1, 287.

PYRAH, L. N., and ALlisON, P. R. (1932), Brit. Med. f., 2 1028

ROSE, S. S. (1950), Brit. F. Surg., in press.

SAMUEL, E. (1950), Brit. F. Radiol., 23, 157.

SWINBURNE, G. (1940), Brit. f. Surg., 27, 713. 